\title{
Enhancing Xanthine Dehydrogenase Activity is an Effective Way to Delay Leaf Senescence and Increase Rice Yield
}

\section{CURRENT STATUS: ACCEPTED}

Rice Springer

Ruicai Han

Jiangxi Agricultural University

$\checkmark$ hrc1988113@163.comCorresponding Author

ORCiD: https://orcid.org/0000-0002-8927-0976

Xunfeng He

Jiangxi Agricultural University

Xiaohua Pan

Jiangxi Agricultural University

Qinghua Shi

Jiangxi Agricultural University

Ziming Wu

Jiangxi Agricultural University

DOI:

$10.21203 / \mathrm{rs} .2 .19763 / \mathrm{v} 1$

SUBJECT AREAS

Plant Physiology and Morphology Plant Molecular Biology and Genetics

KEYWORDS

rice (Oryza sativa L.), xanthine dehydrogenase, allantoin, reactive oxygen species, senescence, yield 
Abstract

Xanthine dehydrogenase $(X D H)$ is an important enzyme in purine metabolism. It is involved in regulation of the normal growth and non-biological stress-induced ageing processes in plants. The present study investigated $\mathrm{XDH}$ 's role in regulating rice leaf senescence.

We measured physical characteristics, chlorophyll content and fluorescence parameters, active oxygen metabolism, and purine metabolism in wild-type Kitaake rice ( Oryza sativa L.), an

OsXDH over-expression transgenic line ( OE9), and an OsXDH RNA interference line ( Ri3 ) during different growth stages. The expression patterns of the OsXDH gene confirmed that XDH was involved in the regulation of normal and abiotic stress-induced ageing processes in rice. There was no significant difference between the phenotypes of transgenic lines and wild type at the seedling stage, but differences were observed at the full heading and maturation stages. The OE9 plants were taller, with higher chlorophyll content, and their photosystems had stronger light energy absorption, transmission, dissipation, and distribution capacity, which ultimately improved the seed setting rate and 1000-seed weight. The opposite effect was found in the Ri3 plants. The OE9 line had a strong ability to remove reactive oxygen species, with increased accumulation of allantoin and alantoate. Experimental spraying of allantoin on leaves showed that it could alleviate chlorophyll degradation and decrease the content of $\mathrm{H} 2 \mathrm{O} 2$ and malonaldehyde (MDA) in rice leaves after the full heading stage. The urate oxidase gene ( UO ) expression levels in the interference line were significantly lower than those in the over-expression line and wild-type lines. The allantoinase ( ALN ) and allantoate amidinohydrolase ( $\mathrm{AAH}$ ) genes had significantly higher expression in the Ri3 plants than the in OE9 or wild-type plants, with OE9 plants showing the lowest levels. The senescence-related genes ACD1, WRKY23 , WRKY53, SGR , XERO1, and GH27 in Ri3 plants had the highest expression levels, followed by those in the wild-type plants, with OE9 plants showing the lowest levels.

These results suggest that enhanced activity of XDH can regulate the synthesis of urea-related substances, improve plant antioxidant capacity, effectively delay the ageing process in rice leaves, and increase rice yield.

Full-text 
Due to technical limitations, full-text HTML conversion of this manuscript could not be completed.

However, the manuscript can be downloaded and accessed as a PDF.

Figures 
(A)

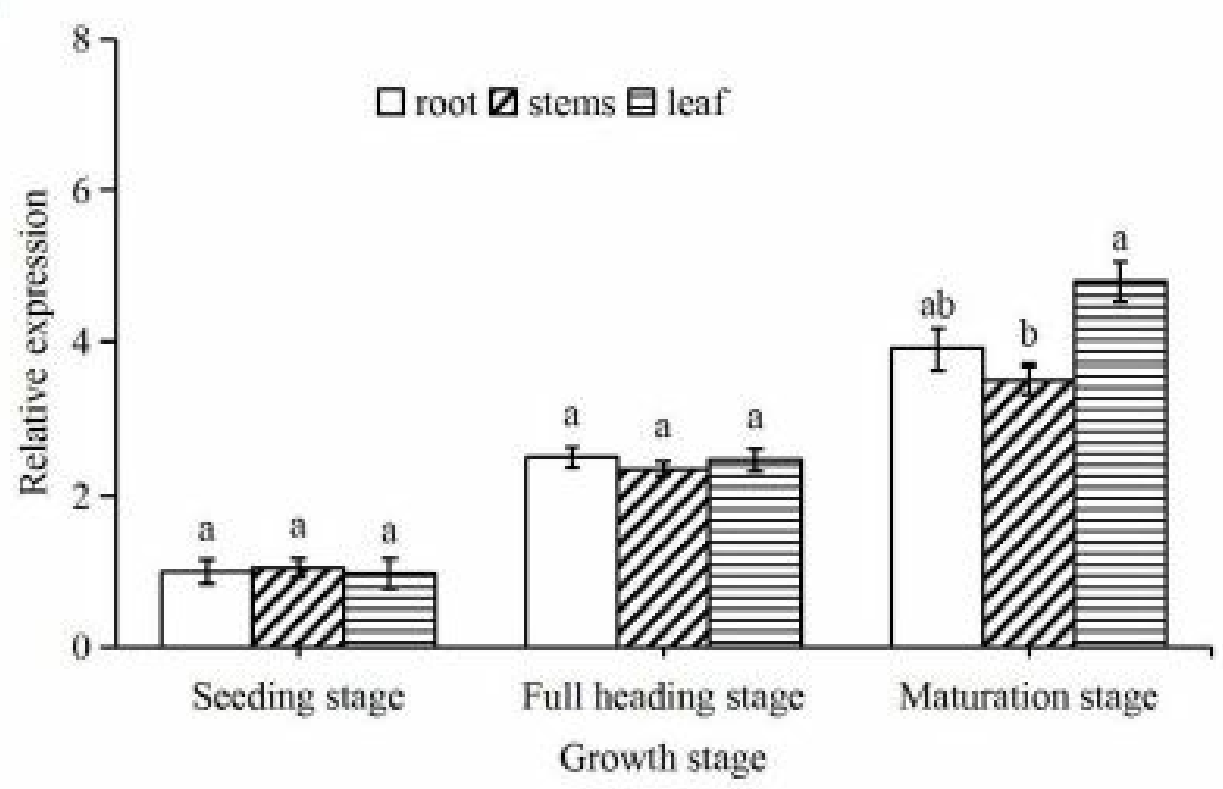

(B)

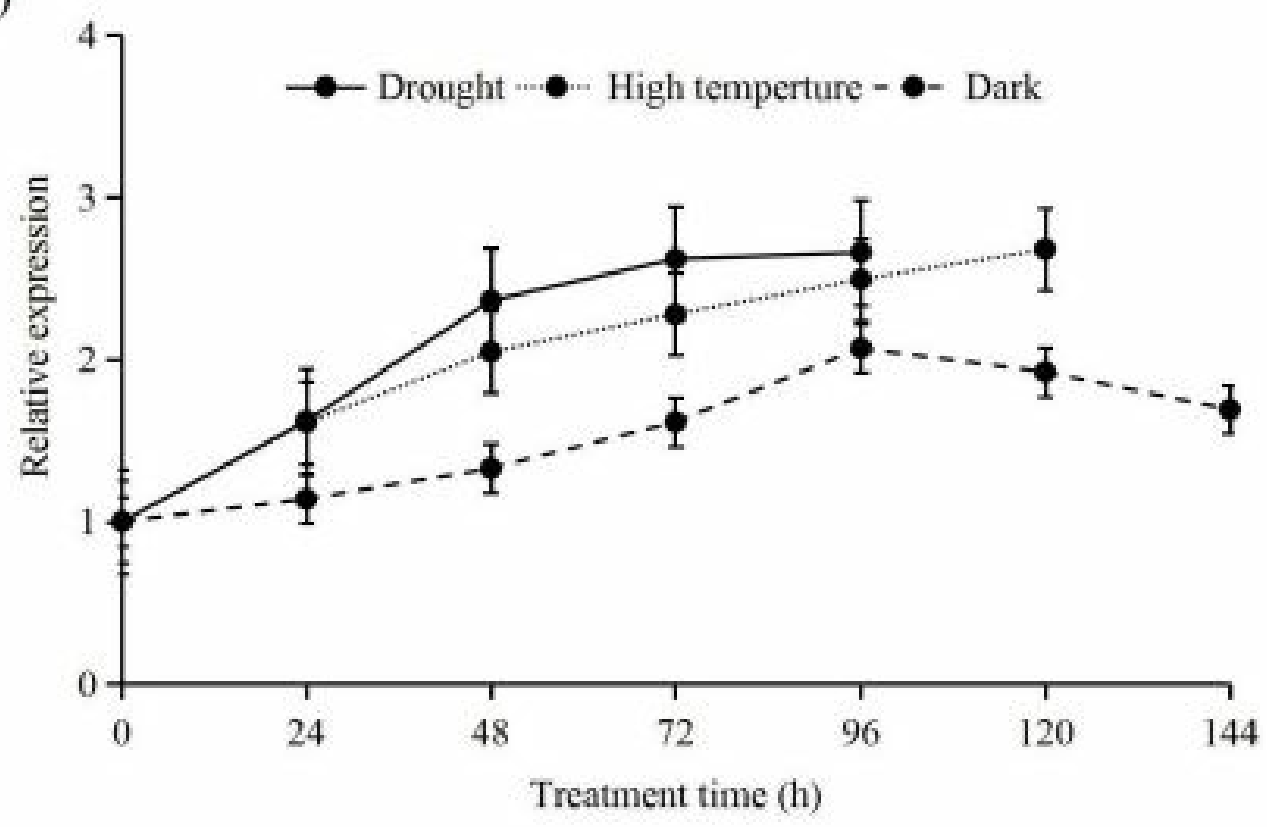

Figure 1

Expression pattern analysis for OsXDH. (A) Expression level of OsXDH in various tissues of rice at different 161 growth stages. (B) Expression levels of OsXDH in rice leaves under different stresses. The column data in figure is average and the short lines represents the mean square deviation, values in the same column show means via the LSD, and different letters indicate that the means were statistically different $(P<0.05), n=3$. 


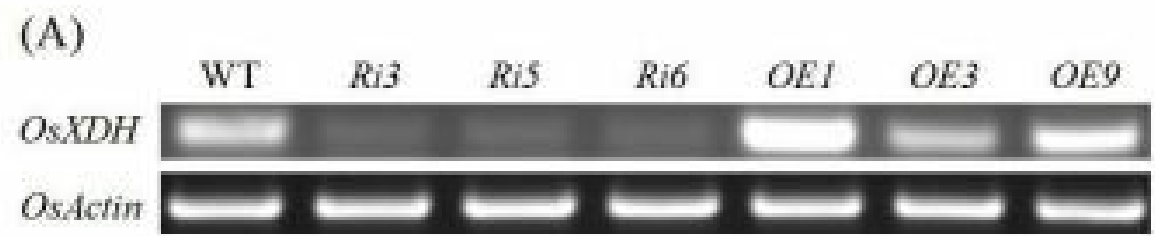

(B)

$$
\text { WT Ri3 Ri5 Ri6 OEI OE3 OE9 }
$$

$\mathrm{XDH}$ activity

Solution protein

Figure 2

The relative expression level of OsXDH and XDH activity in wild-type and transgenic plants.

(A) Expression analysis of OsXDH. The internal reference gene was OsActin. (B) Enzyme activity analysis of XDH. Soluble protein was extracted from rice seedling leaves grown for 30 days. Each lane in the gel was loaded with equal content soluble protein. XDH activity was detected in gel with hypoxanthine as substrate. 


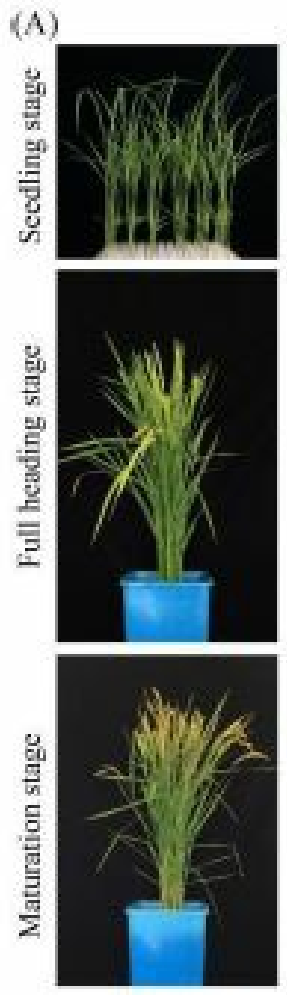

WT
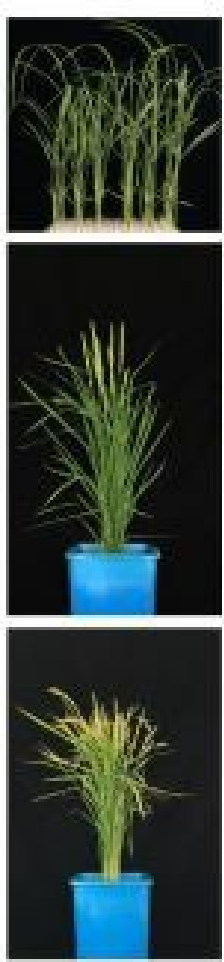

$R i 3$
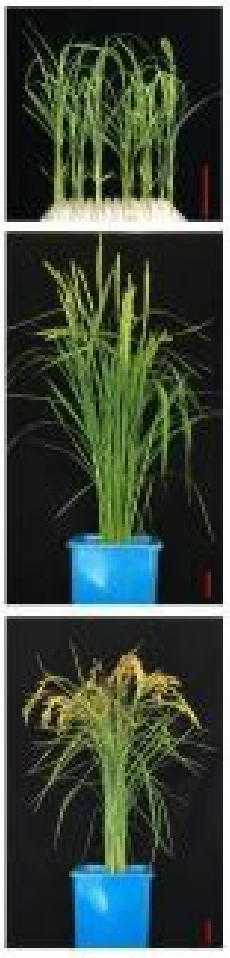

OE9
(B)

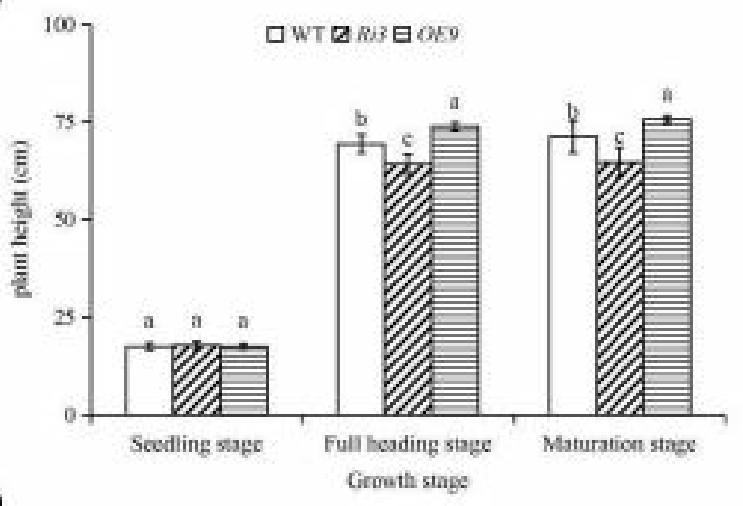

(C)

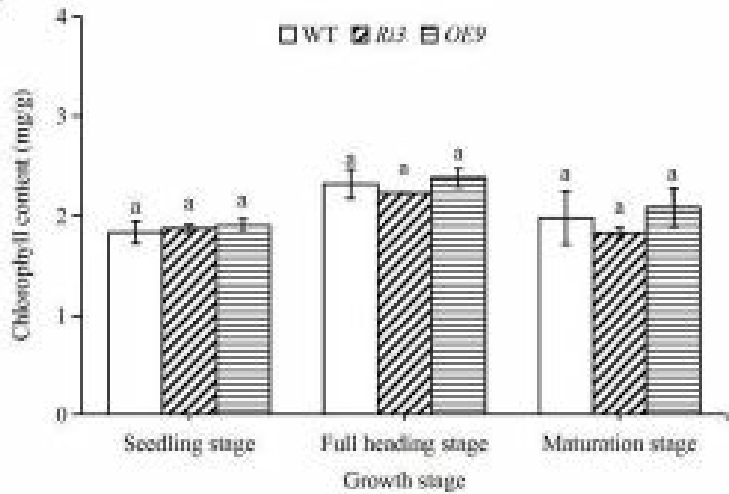

Figure 3

Plant height and chlorophyll content differences between wild-type and transgenic plants at different growth stages. (A) Appearance, (B) plant height, and (C) chlorophyll content of WT, Ri3 and OE9 at different growth stages. The column data in figure is average and the short lines represents the mean square deviation, values in the same column show means via the LSD, and different letters indicate that the means were statistically different $(P<0.05), n=$ 5(B) and 3(C). 
(A)

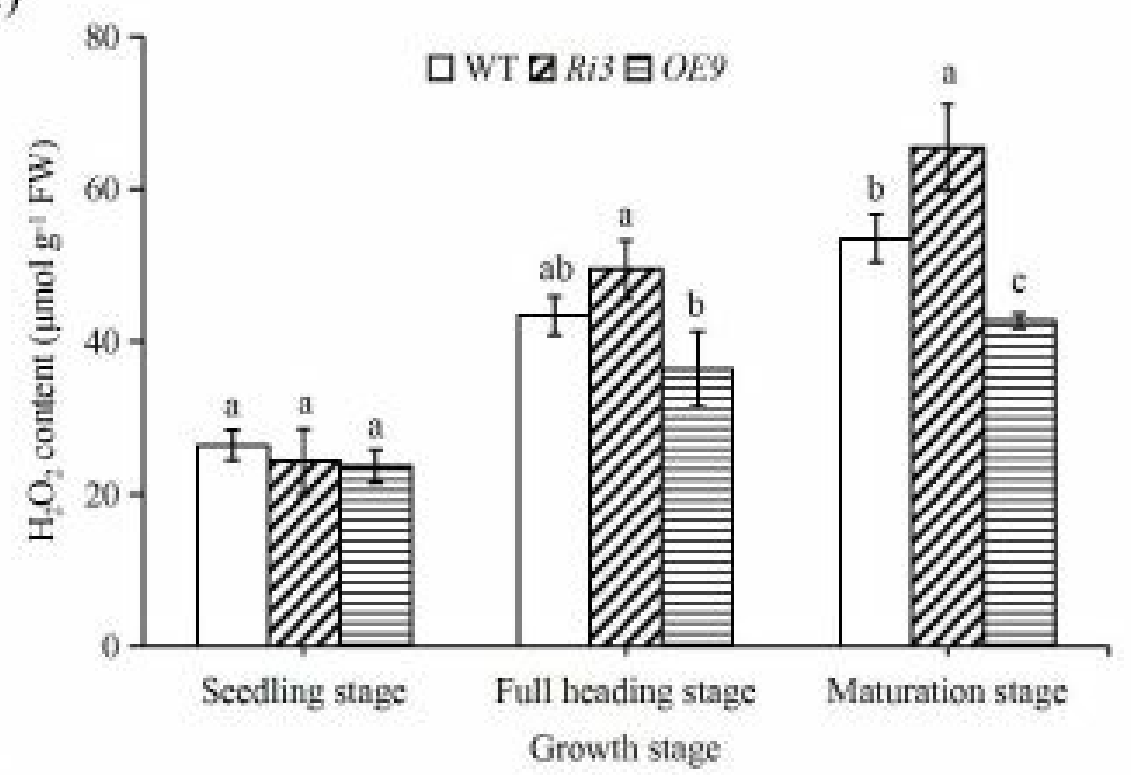

(B)

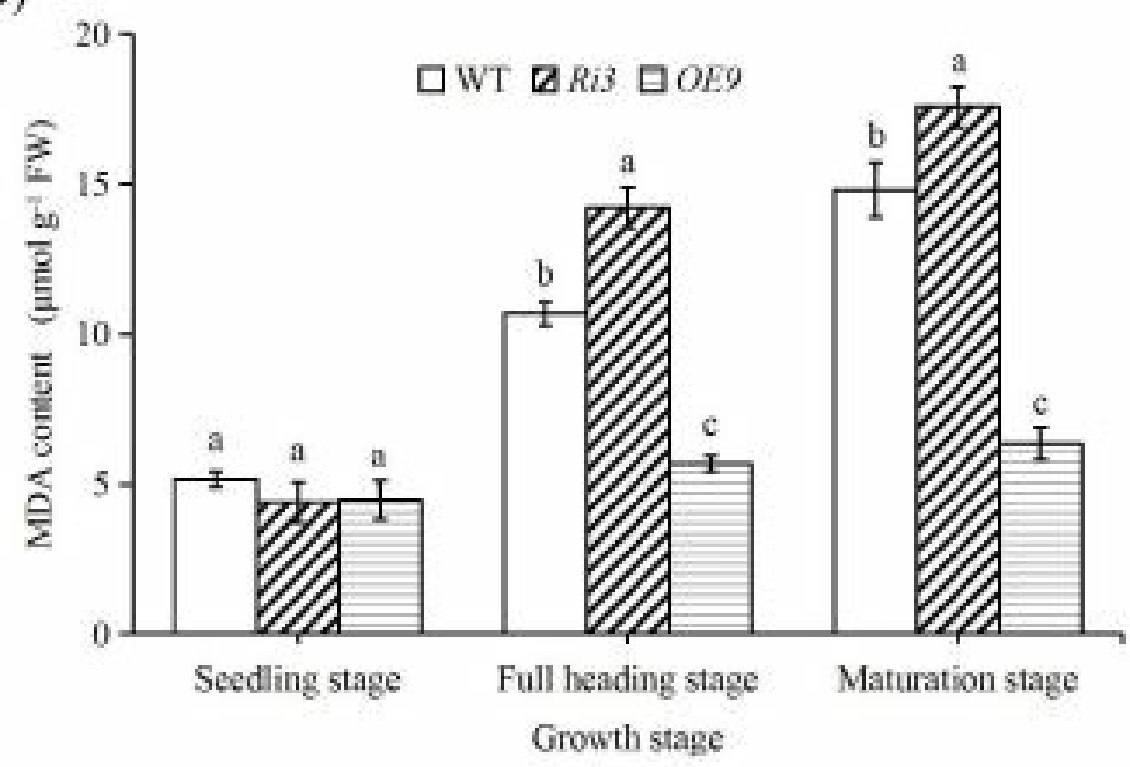

Figure 4

Differences in reactive oxygen metabolism in wild-type and transgenic lines at different growth stages. (A) $\mathrm{H} 2 \mathrm{O} 2$ content. (B) MDA content. The column data in figure is average and the short lines represents the mean square deviation, values in the same column show means via the LSD, and different letters indicate that the means were statistically different $(P<0.05), n=3$. 
(A)

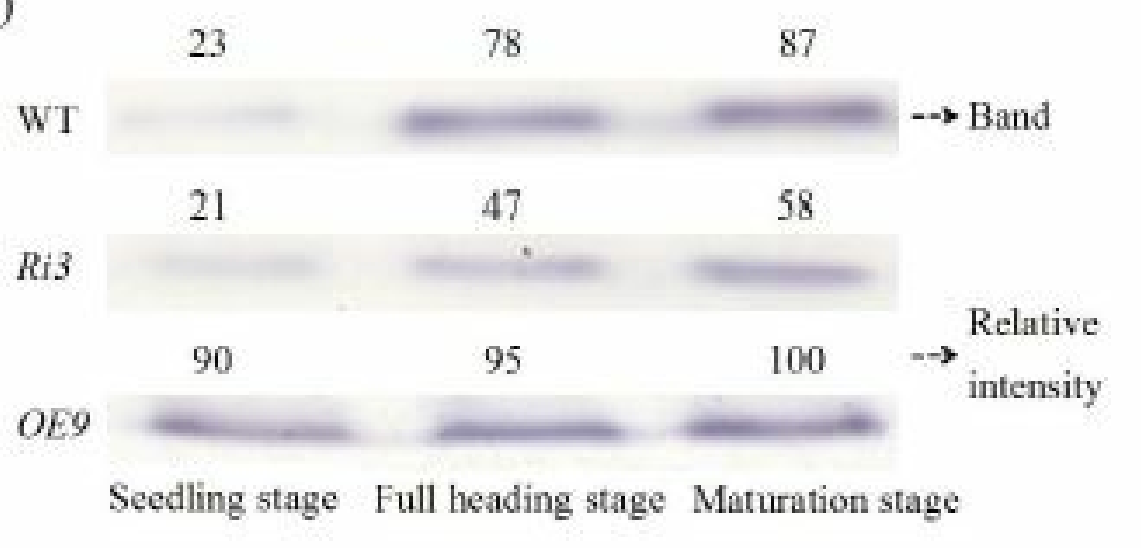

Figure 5

XDH enzyme activity in wild-type and transgenic lines at different growth stages. Soluble protein was extracted from leaves of transgenic plants at different growth stages. Each lane in the gel was loaded with equal content soluble protein. XDH activity was detected in gel with hypoxanthine as substrate. Numbers above the lanes indicate relative intensity obtained by scanning the formazan bands with a computing laser densitometer using Image J 2x software. 


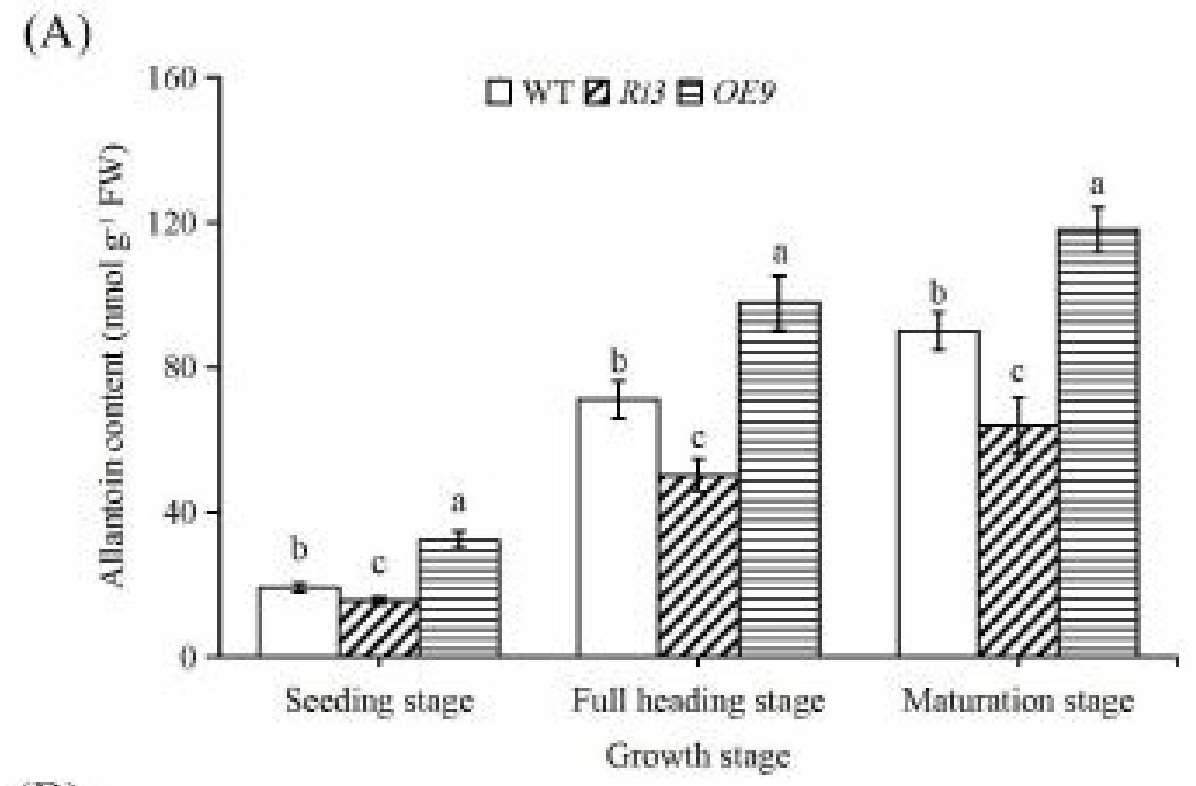

(B)

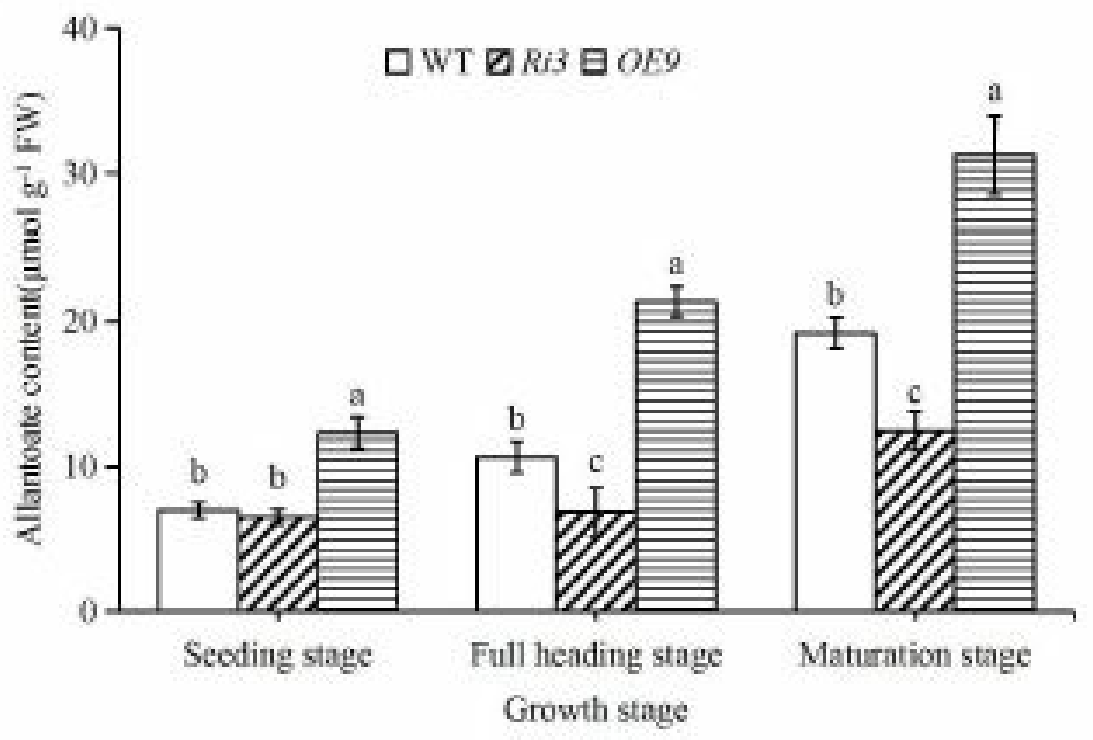

Figure 6

Allantoin and allantoate content in wild-type and transgenic lines at different growth stages. (A) Allantoin content. (B) Allantoate content. The column data in figure is average and the short lines represents the mean square deviation, values in the same column show means via the LSD, and different letters indicate that the means were statistically different $(\mathrm{P}<$

$$
0.05), \mathrm{n}=3 \text {. }
$$


(A)

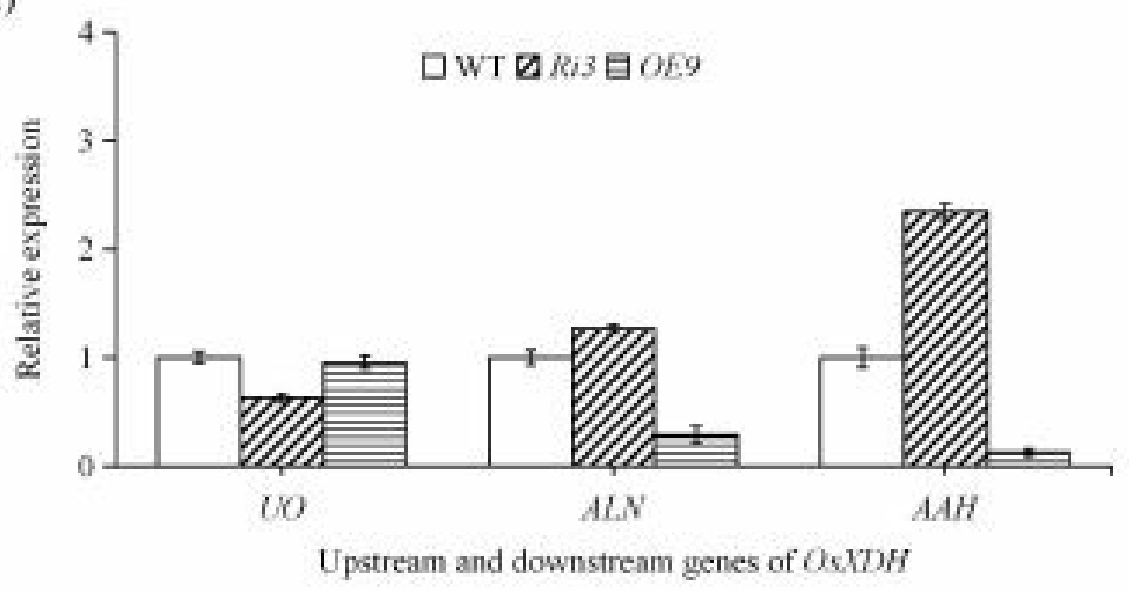

(B)

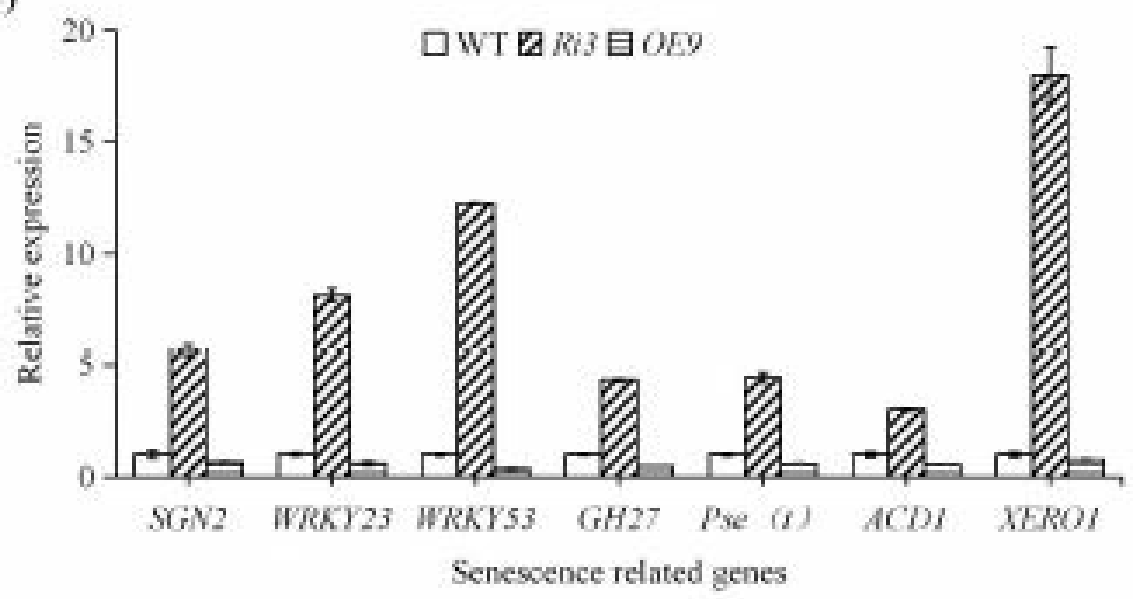

Figure 7

Expression analysis of genes upstream and downstream ofOsXDH and of senescence related genes at the maturation stage. (A) Upstream and downstream genes of OsXDH. (B) Senescence related genes. Ubi served as an internal control. The column data in figure is average and the short lines represents the mean square deviation, values in the same column show means via the LSD, and different letters indicate that the means were statistically different $(P<0.05), n=3$. 


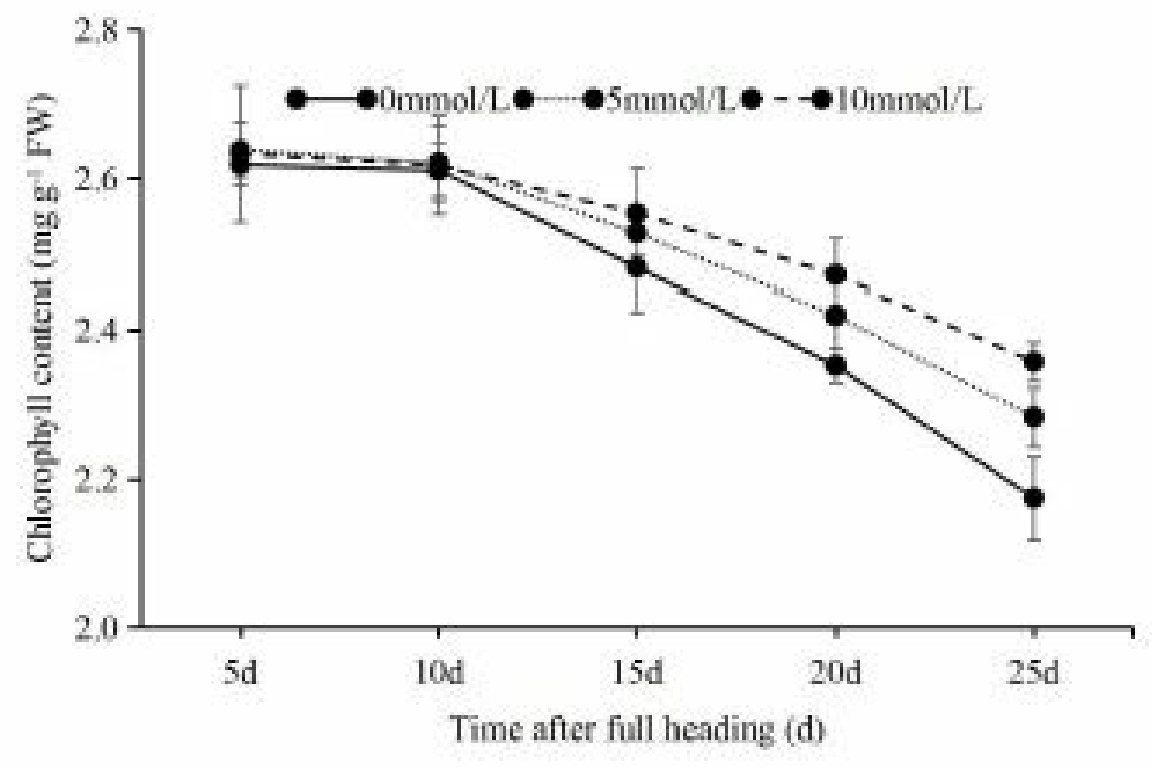

Figure 8

Chlorophyll content of rice after exogenous spraying of allantoin at different concentrations after full heading. The column data in figure is average and the short lines represents the mean square deviation, values in the same column show means via the LSD, and different letters indicate that the means were statistically different $(P<0.05), n=3$. 
(A)

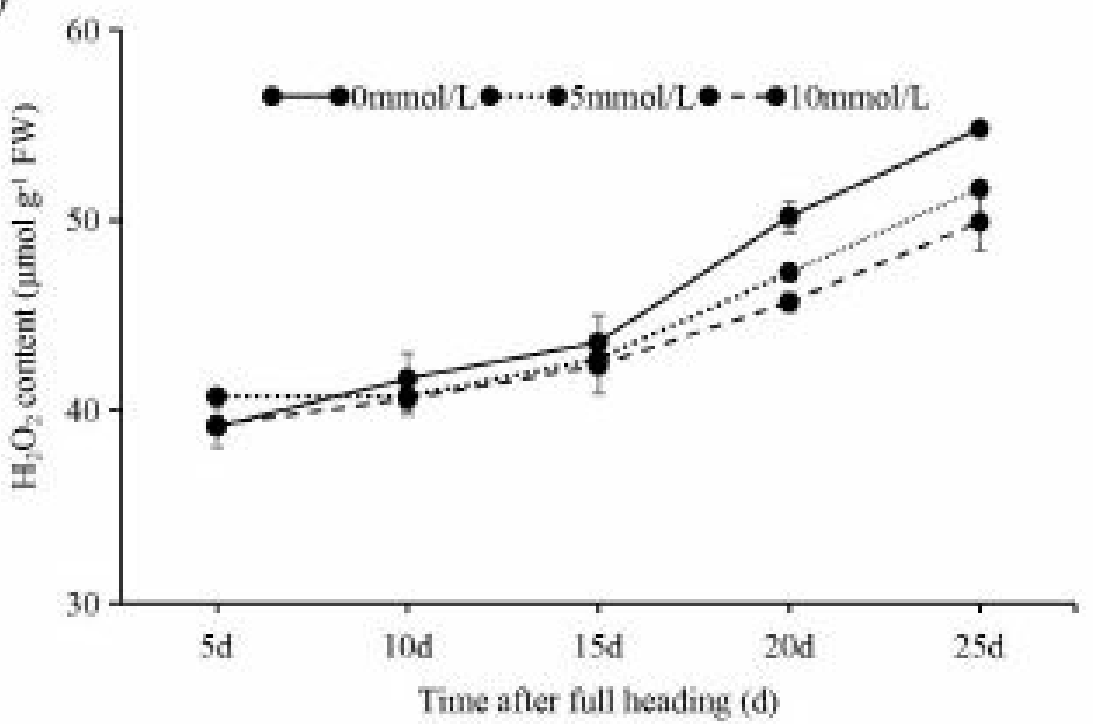

(B)

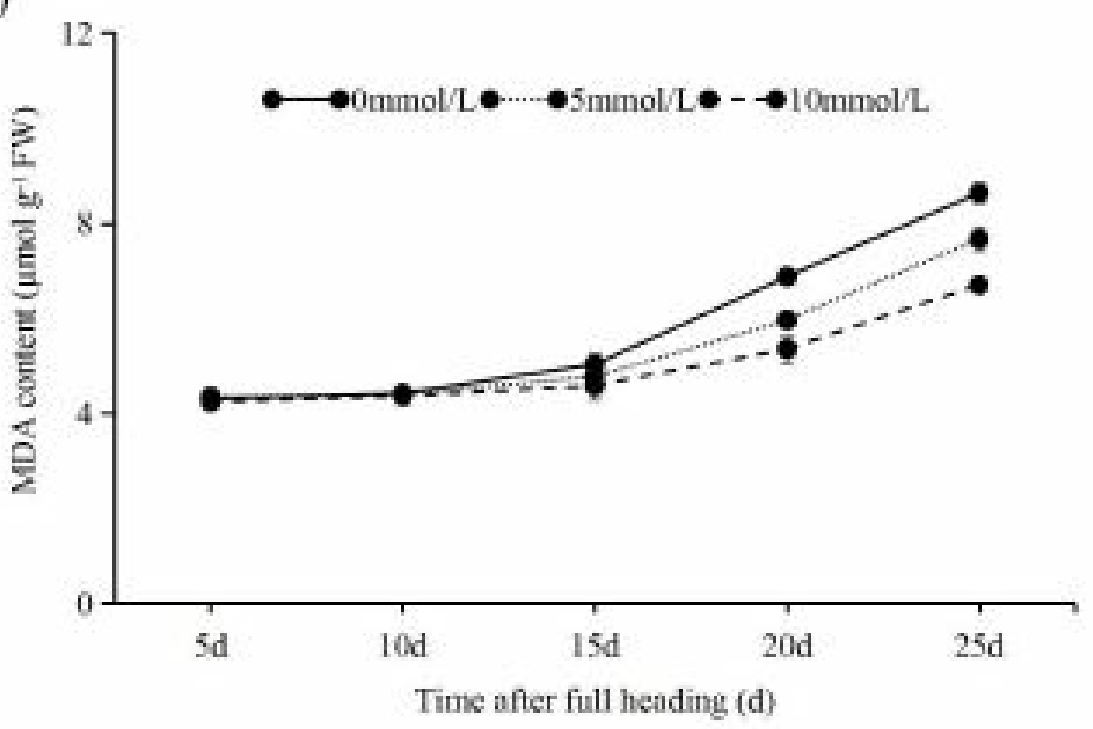

Figure 9

H2O2 and MDA content of rice leaves after exogenous spraying allantoin at different concentrations after the full heading stage. (A) H2O2 content. (B) MDA content. The column data in figure is average and the short lines represents the mean square deviation, values in the same column show means via the LSD, and different letters indicate that the means were statistically different $(P<0.05), n=3$.

\section{Supplementary Files}

This is a list of supplementary files associated with this preprint. Click to download. 
Supplementary Material.pdf 